\title{
Factors influencing women's choice of place of delivery in urban and peri urban areas of Gondar Town, North West of Ethiopia
}

\begin{abstract}
Background: In Ethiopia, antenatal care $34 \%$ delivery service by skilled attendant 10\%, postnatal care $7 \%$. There is now widespread consensus that a principal effective intervention for reducing maternal mortality is the universal use of skilled birth attendants based in functioning health care facilities but still health facility deliveries in the country is very low.

Methods: This explorative study was conducted in Gondar town with investigating individual. Community and health institution factors influencing women's choice of place of delivery. Data were collected through focus group discussion with women and in depth interview with health works. A total of 10 focus group discussions were conducted ( 5 from urban and 5 peri-urban) with women those delivered at home and health institutions. All focus group discussions were tape recorded and transcribed in full. Also twenty in depth interviews were conducted, 10 with midwives those worked in health centers and hospital in antenatal and delivery unit, and 10 with health extension workers worked in health posts at the community level.
\end{abstract}

Results: Almost all participants had antenatal care from governmental health institution but majority of women didn't mentioned pregnancy related complications and the advantage of having skilled attendant at delivery due to constant failure of health service provider in provided information on possible pregnancy related complications, birth preparedness and emergency readiness in health institution and at community levels for both Peri urban and urban women, had no good attitude towards women in labor, unnecessary referral from health center to hospital, lack of competency in diagnosed and management of labor and delivery related complications, high number of operation at teaching hospital, fear of acquiring HIV/AIDS from health institution, lack of privacy, financial constraints for hospital delivery services, transportation problems cultural believes were common factors.

Conclusion: The most important factors influencing women's in the study area choice of place of delivery fall in to quality of care in the health systems itself, particularly lack of communication of health service providers on information about possible pregnancy related complication, the advantage of having skilled attendant at delivery, attitude of health service provider, shortage of well trained staff in midwifery skill, lack of linen and washing facilities, fear of operation, financial constraint for hospital delivery and transportation.

Keywords: health, peri-urban, delivery, antenatal
Volume 2 Issue 3 - 2015

Mulunesh Biweta

Department of Midwifery, University of Gondar, Ethiopia

Correspondence: Mulunesh Biweta, Department of Midwifery, University of Gondar, PO. Box .196, Ethiopia, Tel 251918220016, Emailmulunesh10@yahoo.com

Received: March 29, 2015 | Published: June 19,2015

\section{Background}

Despite several decades of global health initiatives focused on maternal health, maternal mortality has proven to be an interactable problem. The Millennium Development Goal indicator of maternal health, the maternal mortality ratio has remained essentially unchanged over the past 15 years with an estimated mean annual decline of $0.4 \%$ since 1990 far short of the progress required to meet the Millennium Development Goal target of $75 \%$ reduction by 2015 . Maternal mortality ratio measures the risk of dying during pregnancy or shortly after delivery and is thus primarily a measure of the safety of childbirth. Ninety-nine percent of maternal deaths occur in the developing world, and sub-Saharan Africa has the highest maternal mortality ratio in the world. ${ }^{1}$

There is now widespread consensus that a principal effective intervention for reducing maternal mortality is the universal use of skilled birth attendants based in functioning health care facilities to diagnose obstetric complications and manage or refer them. Facility delivery rates in sub-Saharan Africa are some of the lowest in the world only $47 \%$ of women delivered in a health facility in 28 subSaharan African countries. ${ }^{2}$

Despite Ethiopia government's policy to support women to deliver in health facilities with the assistance of skilled attendants many women did not access this care. The maternal mortality ratio of the country suggests that it has actually statistically increased over the past years, and consider ability from 673/100000 in 2005 to almost 676/100000 live births in 2011. Large proportion of deaths occurs during the first 24 hours of postpartum period due to lack of skilled birth attendant and early postnatal care. ${ }^{3}$

Antenatal care $34 \%$ delivery service by skilled attendant $10 \%$, postnatal care $7 \%$, and unmet need of family planning is $32 \%$. The life time risk of maternal death is 1 in 16 , which contrasts to the world average at 1 in 74 and especially to the life time risk industrial countries at 1 in $4,085 .^{3}$ 
Even though the accuracy of the number is uncertain the difference is striking access to professional health care during delivery is considered to critical for maternal mortality reduction. Early identification and proper management of obstetric complication is fundamental for life saving. The antenatal screening has such a low predictive value of identifying risk cases that the presence of a skilled attendant at delivery more likely reduces Mortality. ${ }^{4}$

The common factors among countries managing to reduce pregnancy related death has the presence of skilled providers attending most of the deliveries. In effectiveness of training traditional birth attendants has drawn attention towards training on professional personnel the proportion of births conducted by a skill attendant has become an indicators for monitoring progress towards reduction of maternal mortality. Therefore, the main objective of this study was assessed factors influencing women's choice of place of delivery.

\section{Methodology}

\section{Study design and site}

Community and health facility based cross sectional qualitative study was conducted from March 2014 to April 2014 in Gondar town. Gondar town is found in North Gondar zone of Amhara regional state of Ethiopia and is located $720 \mathrm{~km}$ North West of Addis Ababa with the total of 315,856 population. ${ }^{5,6}$ Administratively the town is divided into 11 peri-urban and 12 urban center towns. The health system in the town is represented by one governmental teaching hospital, eight health centers 23 health post and five higher, twenty two medium and nineteen lower clinics owned by private sectors and 2 NGO's clinics but maternal health care of the town still low.

\section{Ethical considerations}

Ethical clearance was obtained from the institutional Review Board of the University of Gondar. Communication with urban center town and peri-urban administrators were made through formal letter obtained from the University of Gondar. After explained the purpose and objective of the study signed and verbal consent were obtained from each study participant.

\section{Sampling}

Five urban centers and 5 peri-urban towns were randomly selected. 10 focus group discussions were conducted (1 from each) with women who gave birth in health institution and home less than 1 year. In each focus group discussion 8-10 participants were participated. The discussion was tape recorded and it was converted to English written word. In-depth interview were conducted with 10 midwives those worked in health centers and hospitals in antenatal and delivery unit, and 10 health extension workers worked in health posts, semi structured close and open ended questionnaires were used for in-depth interview.

\section{Data processing and analysis}

All of the Focus group discussions were tape recorded and transcribe in full. The transcription were analyzed in an open way using content analysis, In-depth interview were analyzed and the narrative qualitative information was organized and integrated according to emerging themes and concept.

\section{Result}

\section{Women's factors}

Almost all participants had antenatal care. Participants were asked about Perception on possible pregnancy related complications, birth preparedness and emergency readiness. Majority of urban women were mentioned the possible complications during pregnancy, labor and delivery, but rare mentioned by peri-urban women. Financial constraint for transportation and hospital delivery services, majority of participants did not have information on the need of birth preparedness and emergency readiness.

Community factor culturally majority of peri-urban women believed that as labor and delivery was a normal phenomenon. Many of them gave birth at their home those were managed by themselves and/or relatives. Some women attended health institution deliveries due to prolonged and difficulty of labor. No woman who gave birth in health posts or supported by health extension workers due to constantly unavailability of health extension workers at community level particularly at night and week end. In peri-urban husbands and neighbours were decided and the community supported women to took to health institution for having skilled attendant at delivery where there were prolonged or difficulty of labor.

Majority of urban participant were delivered in health institution and some of them were home deliveries but there was no delivery service in the health post. The decision was made by the women, the family to have health institution deliveries. The community supported women in labor to take to health institution when labour was started.

All most all focus group participants mentioned the main cause for not having health institution deliveries were difficulty of getting transportation particularly during the night time, difficulty of road for vehicle, unnecessary referral from health center to hospital, attitude of health service providers, lack of competency health service provider in diagnosed and managed labor and delivery related complications, fear of forced operation, lack of privacy, inconvenient delivery coach and fear of HIV/AIDS were common factors for didn't have health institution deliveries of the women.

\section{Health institution factors influencing women's choice of place of delivery}

In-depth interview with Health extension workers worked in urban town they said that as their had no extra midwifery clinical training skill in managing labor and delivery even they had not delivery set for managing emergency deliveries. Majority of them were new due to rapid turnover with no experience on communication with the women. They did some home visit from Monday to Friday but they couldn't find them because of many of them were governmental workers, small scaled traders and daily laborers.

Peri-urban trained health extension workers were rapidly turn over and engaged by other activities with series difficulty to provide home delivery in scattered households with no transportation. They had no residences nearby health posts. They could take supplies and equipment from respected health centers. Also midwives were interviewed on availability of trained health person with midwifery skill in health center and hospital.

In majority of health centers there were serious shortage of midwives. By didn't have adequate number of midwives, nurses were assigned to manage labor and delivery particularly during the night time that could lead to lack of confidence. Even the available midwives many of them were diploma graduates those need further study in midwifery skill. Some of them would like to leave their professional works for better salary.

The available BSC graduate single midwife in some health center was assigned to provide $\mathrm{F} / \mathrm{P}$, antenatal care and delivery services. They mentioned as they had shortage of time to teach all pregnant mothers those came for antenatal care on possible pregnancy related 
complication, birth preparedness and emergency readiness properly. In health centers there were shortages of equipment, no linen or towel, facilities for washing, and autoclaves in delivery room. In teaching hospital midwives were asked and recorded document reviewed on number of caesarean section the result was $38 \%$ during the study period.

\section{Discussion}

Lack of perception about the pregnancy related complications leading to having low skilled birth attendant. Inspite of having high antenatal care at health post at community level and midwealth center and hospital majority of focus group participants didn't mention about pregnancy related complication. It showed that as health service provider did not provide proper information on regular bases on pregnancy related complication, the need of having skilled attendant at delivery, birth preparedness and emergency readiness at the community and health institution level. According Ethiopia and health survey women who did not deliver at a health facility were asked the reasons they did not deliver in a health facility 61 present stated that a health facility delivery was not necessary and 30 percent stated that it was not customary.?

Study done in Tanzania women's preferences for a home birth and lack of planning for delivery are reinforced by the failure of health care providers to consistently communicate the importance of skilled delivery and immediate post-partum care for all women during routine antenatal visits.

The study showed that as peri-urban women in labor had no power in decision making to have health institution deliveries. Qualitative research done on women's perception of home birth in two rural medical districts in Burkina Faso the findings show that homebirths are frequent because of, lack of decision making power to reach health facilities and some of their ladies feel they're going to have an operation for a minor problem. ${ }^{9,10}$ Health service provider should have good attitude toward women in labour. Participants were mentioned as majority of health service providers had no good attitude to women in labor. More than one informant detailed outright physical abuse by health workers. Perceptions of "harsh" medical staff lead many to choose the comfort and privacy of their own homes for childbirth rather than opt for a skilled attendant. ${ }^{11,12}$ There were Rapid turnover of midwives and health extension workers for education and better salary leading to series shortage of midwives, some of them did not have good attitude, lack of competency, unnecessary referral from health center to hospital and lack of confidence managed labor and delivery.

\section{Conclusion}

i. Women did not recognize risk of pregnancy.

ii. Community has no great awareness on possible pregnancy related complications and the need of having skilled attendant.

iii. Rapid turnover of health extension workers those were worked near by the community.

iv. They did not live nearby and available constantly in health post.

v. They had not adequate skill in managing labour and delivery at in peri-urban.

vi. Difficulty of health extension workers for home visiting and attended home delivery in scattered peri-urban. vii. All focus group participants had antenatal follow up in health institution but they didn't have adequate information on possible pregnancy related complication, birth preparedness and emergency readiness.

viii. There was shortage of midwives in health center.

ix. Some health institution health workers had no good attitude in helping women in labour.

$\mathrm{x}$. Lack of linen and washing facilities in health center.

xi. Lack of autoclave for delivery services in health institution.

xii. Unnecessary referrals from health center to hospitals.

xiii. Lack of privacy particularly in hospital.

xiv. Inconvenient delivery coach.

xv. High number of Caesarean section.

xvi. Cost for hospital delivery services.

xvii. Recommendation

xviii. Provide information to women about the possible pregnancy related complication, birth preparedness and emergency readiness.

xix. Create community awareness on possible pregnancy related complications and the need of having skilled attendant.

xx. To Gondar town health office

xxi. Design strategies and implementation for retention of health extension workers.

xxii. Peri-urban health extension workers should live nearby and available constantly in health post.

xxiii. Provide training for peri-urban health extension workers to have adequate knowledge and skill in managing normal labour and delivery.

xxiv. Health extension workers should not be over loaded by other activity to be able attend home delivery in scattered peri-urban population.

xxv. Health extension workers must have home to home visit on Saturday and Sunday when majority of the community at in their home.

xxvi. Assign adequate midwives in health center.

xxvii. Try to have linen and washing facilities particularly in health centers.

xxviii. Equip facilities with necessary instruments and supplies.

xxix. Strengthening health center delivery services to avoid unnecessary referrals to hospitals.

xxx. Maintain privacy in hospital during labour and delivery.

xxxi. If possible try to change discomfort delivery coach.

xxxii. Minimize high number of operation.

xxxiii. Free of charge particularly for hospital delivery.

xxxiv. The hospital tries to find a means to allow a single family to be with woman in labour. 
xxxv. Midwives and health extension workers work jointly.

\section{Acknowledgments}

None.

\section{Conflicts of interest}

The authors declare there is no conflict of interests.

\section{References}

1. WHO, UNICEF, UNFPA, The World Bank. Maternal Mortality in 2005. World Health Organization, Geneva, Switzerland; 2007.

2. Donnay F. Maternal survival in developing countries: what has been done, what can be achieved in the next decade. Int J Gynaecol Obstet. 2000;70(1):89-97.

3. Central Statistical Agency (Ethiopia) and ORC Macro. Ethiopia Demographic and Health Survey 2005. Addis Ababa, Ethiopia and Calverton, Maryland, USA; 2006.

4. Central Statistical Agency (Ethiopia) and ICF International. Ethiopia Demographic and Health Survey 2011. Addis Ababa, Ethiopia and Calverton, Maryland, USA; 2012.

5. Central statistical authority. The 1994 population and housing census of Ethiopia result for Amhara region. Volume II Part III Statistical report on Migration Fertility and Mortality; 1995.
6. Federal Democratic Republic of Ethiopia Population census commission. Summary and statistical report of the 2007 population and housing census, Population size by Age and Sex. United Nations Population Fund, Addis Ababa, Ethiopia; 2008.

7. United Nations Population Fund (UNFPA). The Cairo consensus at 10: Population, reproductive health and the global efforts to end Poverty. United Nations Population Fund, State of the World Population; 2004.

8. Magoma M, Requejo J, Campbell OM, et al. High ANC coverage and low skilled attendance in rural Tanzanian district: a case for implementing a birth plan intervention. BMC Pregnancy Childbirth. 2010;10:13.

9. Jasper C, Chike O. Traditional Belief Systems and Maternal Mortality in a Semi-Urban Community in Southern Nigeria. Afr J Reprod Health. $2001 ; 5(1): 75-82$

10. WHO, ICM, FIGO. Making Pregnancy Safer: The Critical Role of the Skilled Attendant. World Health Organization, Geneva, Switzerland; 2004.

11. Ronsmans C, Graham WJ. The Lancet Maternal Survival Series steering group. Maternal mortality: who, when, where, and why. Lancet. 2006;368(9542):1189-1200.

12. Seljeskog L, Sundby J, Chimango J. Factors influencing women's choice of place of delivery in rural Malawi expletory study. Afr J Reprod Health. 2006;10(2):66-75. 